\title{
A system for controlling the light output of a monochromator by any simple function and for temporally modulating the intensity 1,2
}

PAUL ROSEN, MICHAEL W. LEVINE, MICHELANGELO ROSSETTO, and I S R A E L A B RAM OV , 3 THE ROCKEFELLER UNIVERSITY, NeW York, New York 10021

This paper describes an apparatus that controls the output of a monochromator so that the energies in each waveband can be equated according to any desired function. $A$ photocell monitors the light from the monochromator and provides a feedback to the power supply driving the lamp. A function generator is mechanically linked to the monochromator's wavelength drive and provides the function for correcting the output spectrum. Once set, the light output remains constant even if the bulb ages or is replaced. If desired, a sinusoidal ripple of variable frequency and amplitude can be imposed on the mean intensity of the light.

Many experiments on vision use either filters or monochromators to control stimulus wavelength. Two problems are involved: (1) equating in some appropriate way the intensities of the different stimuli, e.g., for photopic luminosity, and (2) keeping constant the intensity of a given stimulus as the light source ages or is replaced. Typically, the E uses some photosensitive device to monitor each stimulus and adjusts voltage to the light source, a frustrating and error-prone procedure. This paper describes a method of obtaining spectral stimuli whose intensities can be equated according to any arbitrary function of wavelength and can be maintained at the prescribed levels. The method has an additional useful feature: The intensity of a given stimulus can be varied in time according to some simple function such as a sine wave.

\section{PRINCIPLE OF OPERATION}

The system will be discussed in terms of a tungsten source whose light is passed through a monochromator, and it is the output of the monochromator that is controlled. Figure 1 shows a general system in schematic form. Light from the tungsten source, $L$, is passed through a monochromator, $M$, part of whose output is diverted by Beam Splitter B onto Photocell $P$. The output of $P$ is amplified by $F$, and the output signal is summed with a reference voltage, $R$; the sum represents an error signal, $E$, at the input of Amplifier A, which then drives the light source. This represents a feedback system designed to reduce $E$ to zero. For example, suppose the reference signal is greater than that from $F$. In this case $A$ acts to increase the lamp's intensity and, in turn, the signal from $P$. The output from $F$ is thereby increased so as to reduce $E$ to zero. Similar events occur when the output from $F$ is greater than $R$. If $R$ is stable the system will maintain a constant light output proportional to the value of $R$; conversely, if $R$ varies in time (e.g., sinusoidally), light output from $M$ will follow the same time course, provided the temporal capabilities of $\mathrm{L}$ are not exceeded.

The following analysis demonstrates that the system acts linearly

$$
L=A E=A(R-L F) .
$$

Expanding the right-hand side of Eq. 1, rearranging terms, solving for $\mathrm{L}$, we obtain,

$$
L=\frac{A R}{(1+A F)}=\frac{R}{(F+1 / A)}
$$

If $A$ is very large, then $1 / A \rightarrow 0$ and $E q .2$ $(A \rightarrow \infty)$

reduces to

$$
\mathbf{L}=\mathbf{R} / \mathbf{F} \text {. }
$$

Note that the light source is an integral part of the feedback loop, so that the

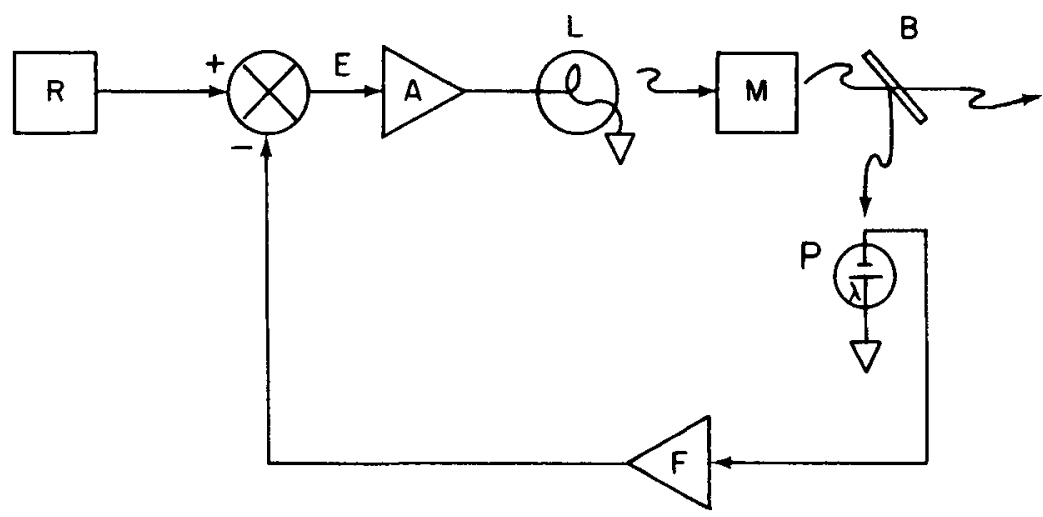

Fig. 1. Block diagram of apparatus. Light from tungsten lamp, $L$, passes through monochromator, $M$; part of the light is diverted by beam splitter, $B$, onto photocell, $P$. Output of $P$ is amplified by $F$ and then summed with reference voltage, $R$. This sum is error signal, $\mathbf{E}$, at output of amplifier, $\mathbf{A}$, which drives lamp. output from $M$ at a given wavelength will always be the same, irrespective of bulb age, etc.; the system will change the output of A until the error signal is nulled or $\mathrm{L}$ fails. This analysis assumes that the output from $P$ is linearly proportional to light falling on it and that given a fixed light intensity the output will remain constant. Obviously the signal from $P$ will depend on $P$ 's sensitivity to the given wavelength of light from $M$. If $F$ is simply an amplifier, inverse of the relative spectral sensitivity of $P$, since the system operates to maintain a fixed output from $P$ when $R$ is constant. However, if the output of $P$ can be multiplied at $F$ by some function of the wavelength of light from $M$, then any emission spectrum can be obtained from $M$. The system for obtaining light output from $M$ proportional to a reference controlling signal from $R$ is described below.

\section{CIRCUIT DESCRIPTION}

The detailed circuit of the scheme discussed above is given in Fig. 2. The reference signal is provided by Amplifier A3. It is the sum of a dc level from Potentiometer R11 (Amplifier A4 minimizes loading of this potentiometer) and a temporal modulation signal from a waveform generator (Model 110, Wavetek, 8159 Engineer Road, San Diego, California; $\$ 450$ ). If temporal modulation is not wanted, simply eliminate the generator. the relative spectral output of $M$ will be the 

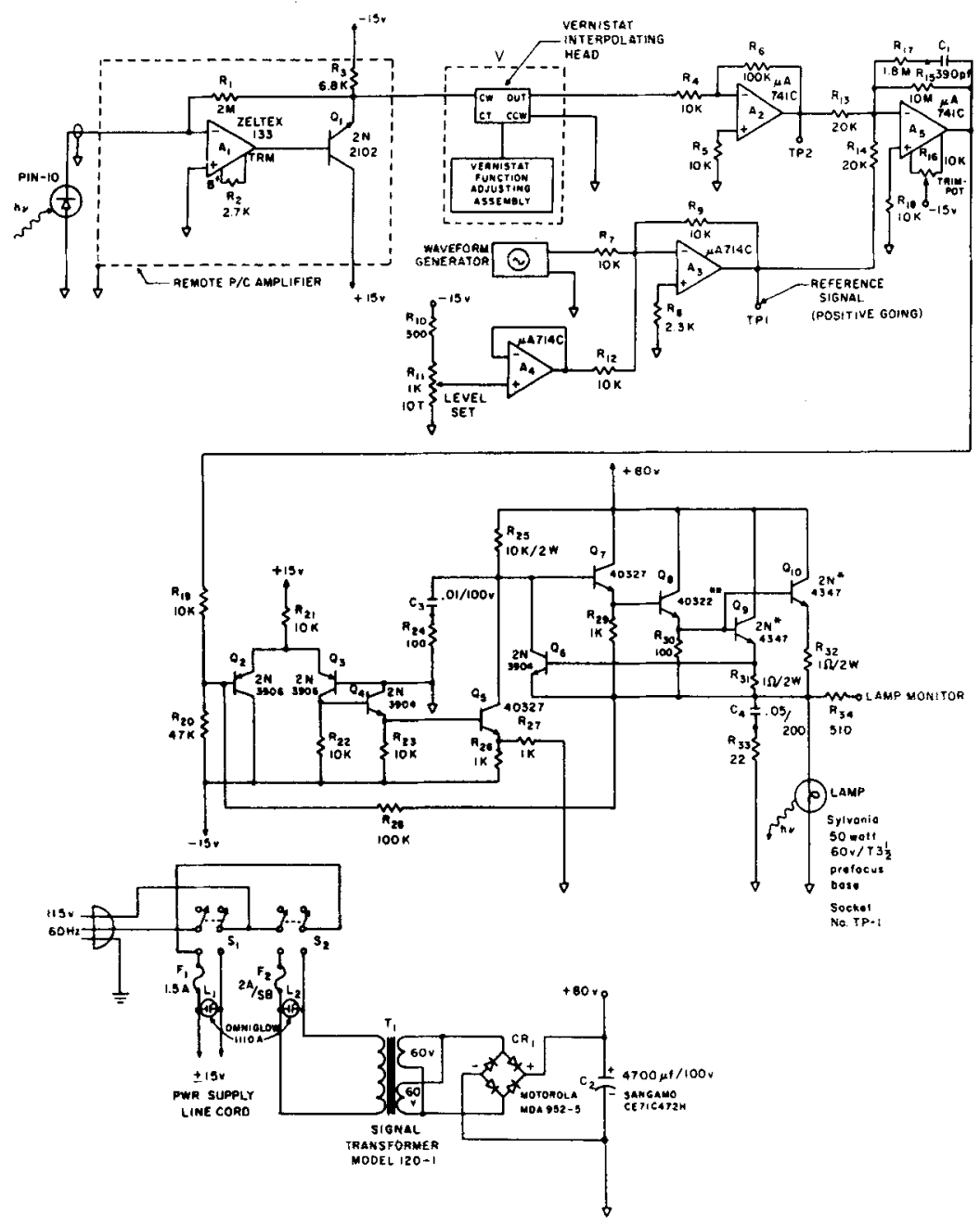

Fig. 2. Detailed circuit of apparatus. See text for details.

Notes:

* Mount on separate heat sinks

**Mount on one of $2 \mathrm{~N} 4347$ heat sinks

1. All resistors in ohms, $1 / 4 \mathrm{~W}, 5 \%$ unless otherwise stated

2. Keep photodiode shielded cable short as possible

3. $L 1$ and $L 2$ indicators are neon lights with series resistor included

4. All capacitors in microfarads unless otherwise stated

The reference signal and inverted feedback signal from Amplifier A2 are summed at $\mathrm{A} 5$, which provides a gain of about 500. This amplified error signal is used as the input signal to an inverting gain-of-10 power amplifier that energizes the lamp. Transistors Q2-Q10 form this amplifier which, together with the gain of A5, is the forward gain, A, in Fig. 1. The $+80-\mathrm{V}$ dc supply voltage for the driver amplifier is obtained from the unregulated supply consisting of transformer, $\mathrm{Tl}$, full-wave rectifier, $\mathrm{CR} 1$, and filter capacitor, $\mathrm{C} 2$.

The light from the lamp is focused on the entrance slit of a monochromator $(1 / 4-\mathrm{m}$ Ebert monochromator, Jarrell-Ash, 590 Lincoln Street, Waltham, Massachusetts; 2-mm entrance and exit slits; $\$ 990$ ) by lens, L1 (Fig. 3). A microscope cover slip at $45 \mathrm{deg}$ to the light path at the exit slit diverts a small percentage of the beam. This side beam is focused by $\mathrm{L} 2$ onto the feedback photocell (PIN-10, United Detector Technology, P. O. Box 5251, Santa Monica, California; \$40). Note that shutters, filters, etc., must be placed after this beam splitter or the feedback system will attempt to compensate for the changes they would produce in light at the detector. The PIN-10 photodiode was selected for its large detecting area ( 0.5 in.), high photosensitivity, and convenient construction; however, any sensitive silicon cell can be used. For improved temperature stability, the photocell is operated in the unbiased, or photovoltaic, mode. The photocurrent developed is fed directly into the inverting input of $\mathrm{A1}$, a FET operational amplifier (Model 133, Zeltex, Inc., 1000 Chalomar Road, Concord, California; \$30). Al provides current through its feedback resistor, R1, to hold its input at virtual ground. Hence, the detector feeds its current into a short circuit. When connected in this way, photocurrent is linearly proportional to incident light intensity. A1, with current booster, Q1, acts as a current-to-voltage converter, with gain proportional to R1. The particular value of $R 1$ will depend on the light output of the monochromator and sensitivity to the detector. It should be chosen such that the voltage to the function generator, $V$, ranges to a maximum of $10 \mathrm{~V}$, just below the saturation voltage of $\mathrm{Al}$.

As pointed out above, the spectral output of the system would be determined by the spectral sensitivity of the photocell if it were not for a modifying function generator, $V$. The modification is performed by a Vernistat Adjustable Function Generator (function adjusting

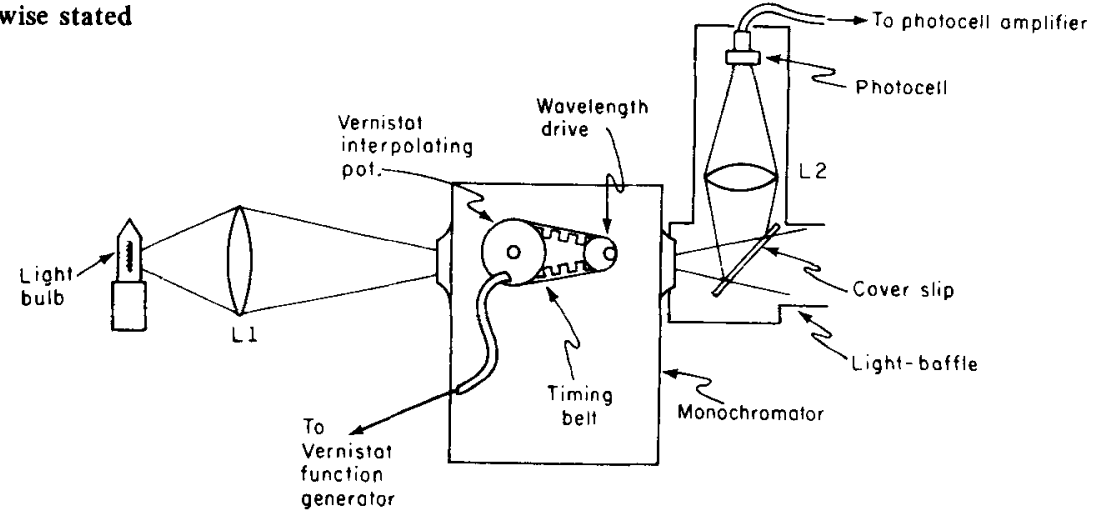

Fig. 3. Simplified form of mechanical and optical system. L1 focuses lamp filament onto entrance slit of monochromator. Microscope cover slip at exit slit diverts part of beam to L2, which focuses light onto photocell. Timing belt rigidly links shafts of interpolating potentiometer (see text) and monochromator wavelength drive. 


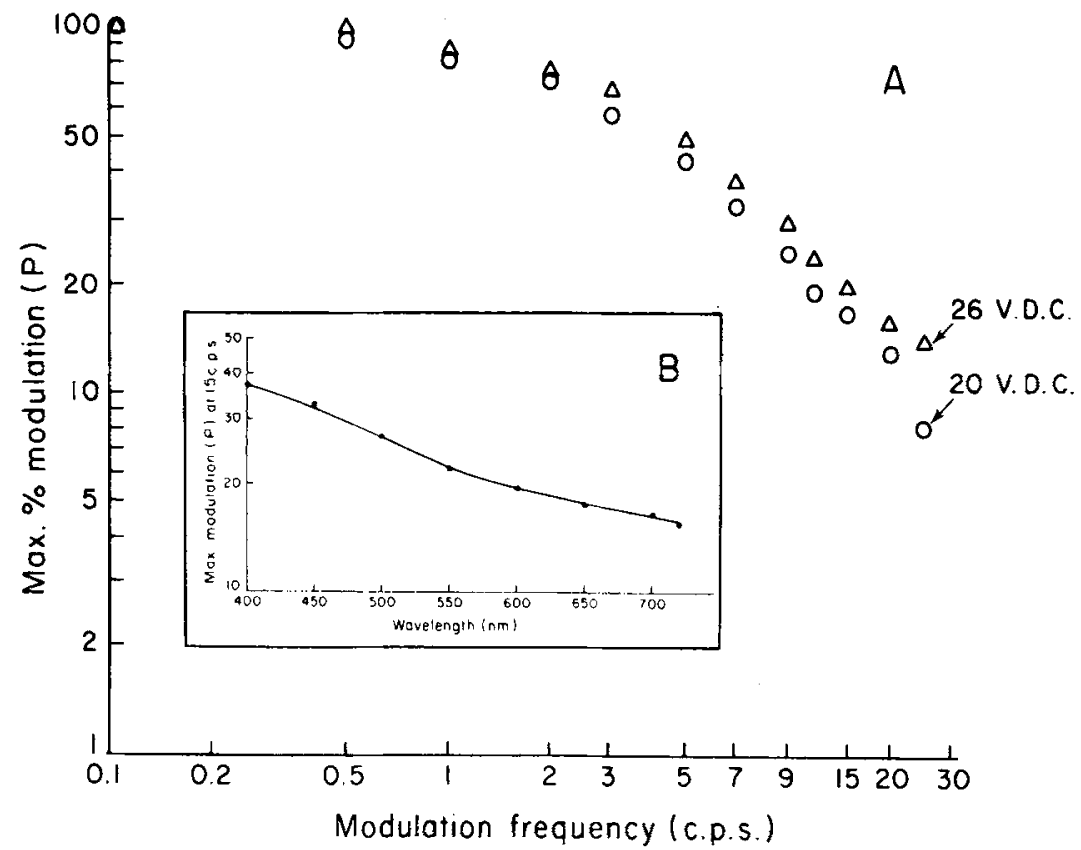

Fig. 4. Maximum sinusoidal modulation that lamp can follow with no distortion and phase shift. Modulation (P) is expressed as percentage of half peak-to-peak amplitude of sine wave to mean light level. A: Frequency response of system. Maximum $P$ as function of modulating frequency (hertz) at two mean voltages. B (inset): Maximum $\mathrm{P}$, at $15-\mathrm{Hz}$ modulating frequency, as a function of wavelength setting. This curve depends heavily on specific optical system and on setting of function generator (see text). For this curve the function generator is set to provide equal quantum flux. Data in A obtained at $570 \mathrm{~nm}$, with same function generator settings as in $B$.

assembly, Model DC250, and interpolating potentiometer, Model 2x5, Perkin-Elmer, Main Avenue, Norwalk, Connecticut; $\$ 900$ ). The function adjusting assembly consists of 34 parallel voltage dividers. Each divider is adjustable to 1 part per 100 by moving its slider along a clearly marked linear track. The interpolating potentiometer is mechanically linked to the wavelength drive of the monochromator, and its shaft position selects the divider used as output. Each divider therefore represents a wavelength setting on the monochromator. When the shaft is at a position intermediate between two dividers, the output is a weighted average of their respective voltages. The spectral sensitivity of the photocell is thus modified according to a 33-secant piecewise linear function of the E's choosing. This function can also include corrections for any wavelength-selective absorptions in the optical train, such as from mirrors and beam splitters. The Vernistat need only be set to produce the desired spectrum as measured at the output of the entire optical system. Thereafter the Vernistat settings for any other spectral function can be obtained by simple calculation, since the function generator operates linearly in the system. In fact, output is proportional to the inverse of the Vernistat setting: To change sensitivity at can be followed perfectly. The criterion for correct servo action is that the system maintain the error signal at null, i.e., no measurable distortion or phase shift between input and output. This is checked by comparing the two inputs, TP1 and TP2, to amplifier A2 (Fig. 2). Note that the response time of the photocell and electronics are entirely negligible in the frequency range over which the lamp can follow.

Choice of light source poses some problems. We used a tungsten lamp, a quartz-iodide type, $50 \mathrm{~W}, 60 \mathrm{~V} / \mathrm{T} 3 \mathrm{H} / 2$-in. prefocused base with mating socket, TP-1 (Sylvania Electric Products, Inc., 60 Boston Street, Salem, Massachusetts; \$15), which provides a satisfactory compromise between emission spectrum, intensity, and temporal characteristics. The lamp has a thin coil filament (about $1.5 \times 4 \mathrm{n}: \mathrm{n}$ : which minimizes its thermal mass, this aiding in rapid response to change in excitation. At its rated conditions, the lamp operates at a color temperature of $2,850^{\circ} \mathrm{K}$. The lamp has a prefocused base, so that position of the filament varies only slightly from bulb to bulb, thereby minimizing optical realignments. In our optical system, it provides a clearly detectable sine-wave ripple on a mean level at frequencies up to $35 \mathrm{cps}$, well beyond the "cut-off" on a "tuning curve" for a human 0 .

For a sinusoid superimposed on a mean level, the ability of the system to servo properly is a function of frequency, mean lamp voltage, wavelength, and percentage modulation, $P$, where $P$ is the percentage of half the peak-to-peak amplitude of the sine wave to the mean. This interdependence can be seen in the "tuning curves" in Fig. 4. The maximum $P$ obtainable at various frequencies of sinusoidal modulation, with no measurable distortion of phase shift, is shown in Fig. 4a. The two sets of points are for the same wavelength but for two different mean lamp voltages. Similarly, ability to follow the driving signal varies with wavelength. As an example, Fig. $4 \mathrm{~b}$ shows the maximum $\mathrm{P}$ obtainable at $15 \mathrm{cps}$ with an equal quantal flux spectrum in our optical system. Naturally, $P$ is greater at lower frequencies, but the exact shape of the curve would change if a different spectrum were used; in general, ability to follow will be better at higher lamp voltages because the filament cools more rapidly at higher filament temperatures.

\section{COST AND SUBSTITUTES}

The approximate prices of the major components were given earlier. The cost of the remaining electronic parts totals approximately $\$ 150$. Not included is a 


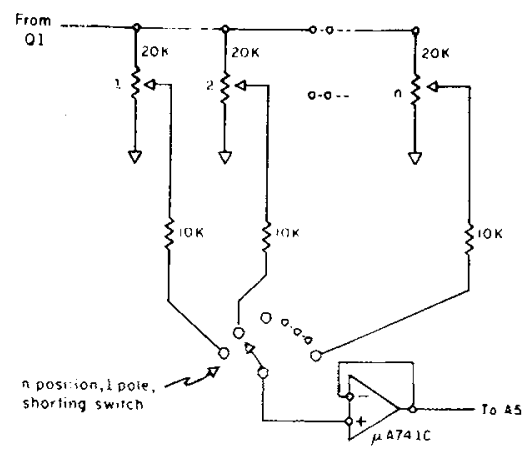

Fig. 5. Alternative form of function generator, replacing $V$ in Fig. 2. See text for details.

$\pm 15-\mathrm{V}$ dc (100-mA) power supply; there are many suitable ones (e.g., Model 2.15.100, Semiconductor Circuits, Inc., 163 Merrimac Street, Woburn, Massachusetts; \$48). This gives a total price, including monochromator and waveform generator, of about $\$ 2,600$. Excluding the monochromator, the most expensive items are the waveform generator (which can be eliminated) and the Vernistat.

If the output will be used only at a discrete set of wavelengths, for instance if filters are used instead of a monochromator, it may be preferable to replace the Vernistat with a set of potentiometers and a switch. The changes from the circuit in Fig. 2 are given in Fig. 5. The potentiometers are in parallel, each one representing a particular wavelength, and the appropriate output is selected by the switch. To avoid loading the photocell amplifier, the parallel impedance of the potentiometers should not be less than $500 \mathrm{ohms}$; up to $3020 \mathrm{~K}$ potentiometers may be used. The $10 \mathrm{~K}$ resistors on each contact are to prevent possible damage during switching; the switch must be of the "shorting" (make-before-break) type to avoid opening the feedback loop and damaging the lamp. The additional operational amplifier is needed to preserve the linearity of the potentiometers by not loading the wipers. Note that if the ratchet is removed from the switch, the device begins to approximate the Vernistat. The output sensitivity will be a step-wise approximation to the desired function. Between potentiometers the output is the mean of the adjacent settings. Therefore, the number of steps will be one less than twice the number of potentiometers.
However, only alternate steps can be set by the E. Furthermore, the sliders on the Vernistat are very conveniently arranged in the form of a graph, with shaft position of the interpolating potentiometer along the abscissa and each slider forming an ordinate.

Finally, the power amplifier in Fig. 2 (Q2 through Q10) may be replaced by a commercial programmable supply (c.g. Model 6824A, Hewlett-Packard, 1101 Embarcadero Road, Palo Alto, California; $\$ 350$ )

It should be emphasized that we have constructed and used the device detailed in Fig. 2; we have not actually tried all the substitutes we suggest in the text, and their use may entail some modifications of the detailed circuit.

\section{NOTES}

1. This work was supported in part by the following grants: EY-00188 from the National Eye Institute, U.S. Public Health Service, GM 01789 from the National Institute of General Medical Sciences, USPHS, and GB 6540 from the National Science Foundation.

2. We wish to thank Dr. Frederick A. Dodge Jr. for many helpful suggestions and criticisms.

3. Address all correspondence to I. Abramov. 